\title{
SPECIAL APPROACH FOR THERMAL MODELLING FIBRE- REINFORCED COMPOSITES WITH LARGER ASPECT RATIO
}

\begin{abstract}
Fundamental solutions and their derivatives located along fibre axes are presented to simulate the interactions of matrix and reinforcing elements in composite materials, when the primary field is a scalar function temperature in heat conduction. The inter-domain continuity is specified in discrete points on fibres boundaries. Intensities of the source functions are defined by ID NURBS (Non-Uniform Rational Basis Spline) and computed in LS (Least Square) sense in the fibres. The inter-domain continuity equations have to be completed by balance equations (energy, equilibrium, etc.) in order to obtain temperature in centre of each fibre. Gradients of temperature are supposed to be constant in cross-sections of the fibres and are computed iteratively by considering them to be linear along fibres in the first step. Material properties of both matrix and fibres are assumed to be homogeneous and isotropic. Three numerical examples giving two fibres overlapping in some length in infinite matrix show the numerical behaviour of the problem for heat conduction.
\end{abstract}

Keywords: MFS, composites, finite fibres, meshless method, source functions, NURBS

\section{Introduction}

In past decades, fibre-reinforced composites have been widely used in engineering applications due to the superiority of their electro-thermo-mechanical properties over the single matrix. Particularly, composite materials reinforced by fibres, or with Carbon Nano-Tubes (CNT) are often defined as materials of future. Although composite materials are designed as fatigue insensitive, especially when compared to metallic ones, they suffer from fatigue loads as well [1 and 2].

Understanding the physical behaviour of these fibre-reinforced composites is essential for structural design. The aspect ratio of fibres (length to diameter) is often very large and all well-known numerical methods, including the Fast Multipole BEM (FMBEM) [3] are not effective to simulate such problems.

The fundamental solution is basis for both the Boundary Element Method (BEM) and the Method of Fundamental Solutions (MFS) [4 and 5]. As for the BEM, elements are used to satisfy boundary conditions and the fundamental solutions (FS) are considered to be continuously distributed along the domain boundaries on elements being one dimension lower than the domain, the MFS is a meshless method using discrete source functions, FS, located outside the domain. Both methods have some advantages and drawbacks, as it is well known [6].

Recently developed Method of Continuous Source Functions (MCSF) [7 and 8] uses 1D continuous source functions - fundamental solutions and their derivatives located along fibre axis to simulate effect of fibres in the matrix, i.e. the interaction of the matrix with fibres in composite material reinforced by the fibres. We present here the problems when the primary field is a scalar function (e.g. temperature in heat conduction) or, if it is a vector function (e.g. displacements in elasticity). The material properties are investigated on a Control Volume Element (CVE) starting with inter-domain conditions on the fibre boundaries corresponding to constant gradient field in corresponding homogeneous field of matrix material and changes in the field are computed due to reinforcing fibres by splitting the problem into two parts, the first one corresponding to the homogeneous material and the second one corresponding to the reinforcing effect.

The inter-domain boundary (compatibility) conditions and basic unknowns have to be specified so that good numerical stability is achieved. It is realized by specifying field quantities (temperatures, displacements, strains) by difference of the value in a collocation point and that in a related point (centre of the fibre, or another point on a corresponding fibre cross-section boundary).

The inter-domain continuity equations have to be completed by balance equations (energy, equilibrium, etc.) in order to obtain temperature, displacement, etc. in the centre of each fibre. The solution is obtained iteratively considering constant value temperature, displacement, etc. along each fibre in the first step. Gradients of the field values define gradients of a similar field in the fibres, both related by material properties of matrix and fibres and they define corrections for inter-domain compatibility in the next iteration step.

The resulting system of equations is evaluated by the LS method. This enables to satisfy the inter-domain boundary conditions in a cross-sectional direction with good numerical stability of the

\footnotetext{
* Milan Zmindak, Pavol Novak

Department of Applied Mechanics, Faculty of Mechanical Engineering, University of Zilina, Slovakia, E-mail: Milan.Zmindak@fstroj.uniza.sk
} 
models. Variables inside the fibres are described by classical methods used in continuum mechanics.

Note that the model assumes that the fields in cross-sections are constant, e.g. only axial stiffness of fibres is considered. As the aspect ratio is large, the bending stiffness of fibres is negligible comparing to their axial stiffness and such assumption reduces the problem, too.

Note also that the cross-sectional dimensions of CNT are very small and methods of continuum mechanics are not applicable for such a problem. However, the axial dimensions are much larger and so the models are applicable also for the material reinforced by CNT.

\section{Source functions in MCSF models}

We assume all matrix materials and fibres are homogeneous and isotropic, the dimensions of the matrix are infinite and models are restricted to linear behaviour. Primary variables can be scalar like in temperature field in heat conduction, or vector like displacement field in deformation of elastic bodies by forces. All the fields are split into two parts, the homogeneous part corresponding to the homogeneous problem of the matrix without fibres and local part containing the influence of interactions of fibres with the matrix. We will deal especially with the local fields in the matrix of the composite material.

The MCSF is a meshless method which satisfies the interdomain compatibility in collocation points (Fig. 1) on the fibrematrix interface.

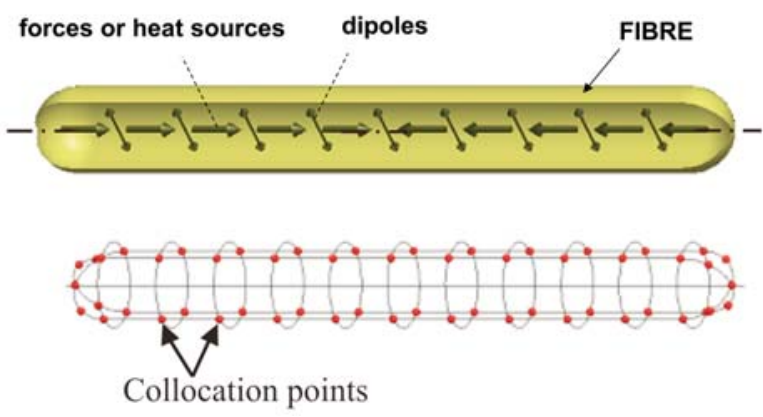

Fig. 1 Distribution of source functions and collocation points

Due to a large aspect ratio of fibres, the stiffening effect in elasticity and conductivity of fibres is realized especially by corresponding effect in the fibre axis direction. Because of this, the inter domain compatibility between the fibre and matrix can be simplified and we assume that the temperature, displacement, strain, stress, etc. in all points of the cross-section are equal to each other.
The interaction of fibres with matrix is simulated in the MCSF by source functions which are 1D-continuously distributed along the fibre axis. The source functions are fundamental solution of the corresponding problem (heat sources in heat conduction and forces in elasticity) and their derivatives. The forces are directed in the fibre axis direction. These source functions, however, are not able to simulate correctly the interaction of fibres with the matrix. In addition the derivatives of the source functions, heat dipoles are included along the fibre axes.

The temperature field induced by a unit heat source acting in an arbitrary point of infinite domain is the fundamental solution for heat problems and it is given by:

$$
T=\frac{1}{4 \pi r}
$$

where $r$ is the distance of the field point $t$ and source point $s$, where the heat source is acting at, i.e.

$$
r=\sqrt{r_{i} r_{i}}, \quad r_{i}=x_{i}(t)-x_{i}(s)
$$

with the summation convention over repeated indices.

Temperature field induced by a unit heat dipole in $x_{i}$ direction is:

$$
T=\frac{1}{4 \pi}\left(\frac{1}{r}\right)_{, i}=-\frac{1}{4 \pi} \frac{1}{r^{2}} r_{, i}=-\frac{1}{4 \pi} \frac{r_{i}}{r^{3}}
$$

and

$$
r_{, i}=\frac{\partial r}{\partial x_{i}(t)}=\frac{r_{i}}{r}
$$

\section{Computational simulation in the MCSF}

In order to find the unknown intensities of the source functions, we have to solve the 1D quasi-singular equations in the form:

$$
\int_{\Gamma} K(s, t) f(t) d \Gamma=g(s)
$$

where $K(s, t)$ is the kernel function which is corresponding to the source function in our case, $f(t)$ is the unknown intensity of the source function, $g(s)$ is the function containing boundary conditions (BC) and $\Gamma$ is the $1 \mathrm{D}$ integration curve along fibres' boundaries.

Note that in the present model the solution of the integral equation (5) is solved in the discrete points, $s$, of the fibre-matrix interface and the integration path and integration points, $t$, are outside of the domain, which is the matrix material. Because of it we can call this method a quasi-meshless method.

In the numerical evaluation of the integral equation (5) the intensity of source functions is approximated by 1D quadratic NonUniform Rational Basis Splines (NURBS) [9] which enable to define shape functions to have continuous first derivative over the 
whole integration path and non-equal distribution of nodal points Because of large gradients in the source functions close to the end parts of the fibres this is a good choice for numerical stability of the model [7 and 8].

Basic variables are temperature in heat and displacement in elasticity problems. Basic source functions are defined by unit heat source in heat and unit force in elasticity. Note that the corresponding source functions for these functions are weak singular for both problems (see equations (1) and (4)).

In the present formulation it is assumed that the fibres are superconductors in heat and rigid in elasticity problems. Moreover for simplicity we consider that the fibres are straight, parallel and constant gradient of temperature and displacement in fibres are considered in the fibre axes direction.

The temperature is not known in the composite, the $\mathrm{BC}$ for the local problem can be specified as follows:

- temperature difference between the corresponding collocation point and the centre of the fibre (it is opposite to the temperature difference in the homogeneous material),

- temperature difference between pairs of points on the opposite sides of the fibre cross-section for heat transfer.

The inter-domain continuity is satisfied using heat sources and heat dipoles with the vector direction perpendicular to each other and also to the fibre axis in heat transfer problems and by unit forces in fibre direction and two force dipoles perpendicular to each other and also to the fibre direction and two couples with torque effect perpendicular to the fibre axis. All these sources are 1D continuously distributed along the fibre axis.

However, temperature change of the centre of each fibre by the interaction is not known a priori in the heat problem. It is obtained by energy-balance/equilibrium condition in each fibre. This is realized by including further r.h.s. by prescribing temperature in corresponding fibre equal to one and zero for the other fibres. In this way we have as many additional r.h.s. as fibres in the problem.

For the finite conductivity of fibres the temperature change along fibre is computed by iteration process starting with superconducting fibre material. One can obtain temperatures and temperature gradients along fibres directly and from distributed source functions the heat flow in the fibre as a result in each iteration step. Because of dimensions the constant heat flow in the cross-section of fibre is a good approximation. From the heat flow and fibre material conductivity one can obtain temperature distribution in the fibre and correct the r.h.s. for the next iteration step.

The problem is very similar in the elasticity [7]. The iteration starts with assuming stiff fibres and prescribing displacement along a fibre without corresponding rigid body displacement in its centre. Displacements, strains and stresses are obtained directly in the matrix and total forces in cross-sections are computed from the source functions. Deformation of fibre is obtained assuming con- stant strains in the fibre direction and for finite stiffness of fibres and corrected r.h.s. is used for the next iteration step.

\section{Computational results}

Figure 2 shows a single straight fibre and patch of parallel fibres regularly distributed and overlapping in some length. To show some rules for modelling the composites with short fibres three examples with only two fibres are presented. The aspect ratio is 50:1, distance between axes of fibres is 4 (all quantities are dimensionless), diameter is 2 , length is 100 . The fibres overlap $\Delta$ in the length 4 for the first two examples and 40 for the third one. The conductivity of fibres is 20000 times larger than that of the matrix in the first example and 1500 times larger in the second and third examples. Temperature gradient is equal to 1 in the fibre axis direction.

Intensities of source functions, heat flow and temperature along fibres are given in Figs. 3 to 5. Figures a) to c) correspond to examples 1 to 3 .

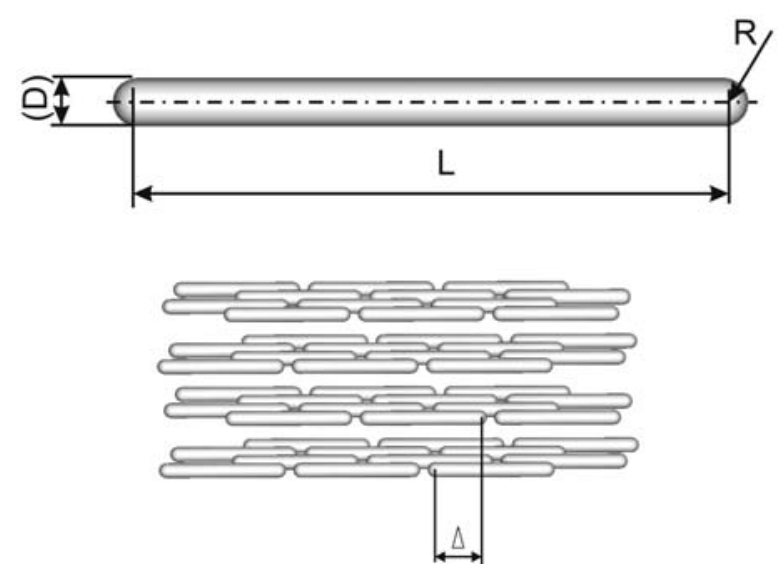

Fig. 2 Single straight fibre and patch of overlapping straight fibres

The red dotted line gives the computed results in the first iteration step, the blue full line is from the last iteration and the green dashed lines for the middle iteration steps. Note that the converged values are obtained in the second iteration for much larger conductivity in fibres to the matrix and in the fifth step for the smaller one with good accuracy.

Note in Fig. 3 the large gradient in source function intensity by the end of fibres but also in the closest points to the end of the neighbour fibre. In those parts it is necessary to choose nodal points defining the source function by NURBS dense. Appropriate density of the nodal points avoids numerical instability of the function. In documented examples the coordinates along fibre are given in Table 1 and are the same for all the examples. 


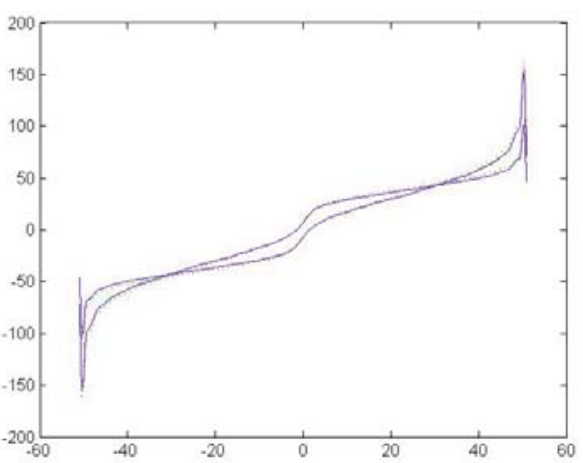

a)

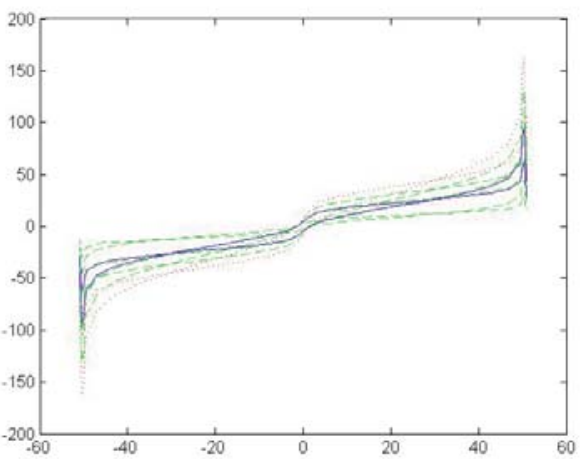

b)

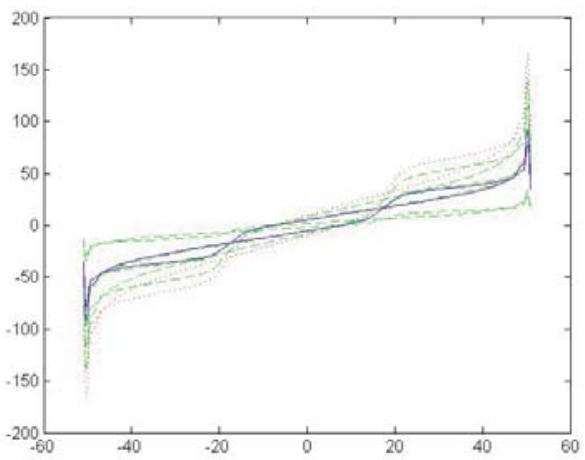

c)

Fig. 3 Heat source intensities along fibres ......., the first iteration step - - - - -,

the middle iteration steps, — the last iteration

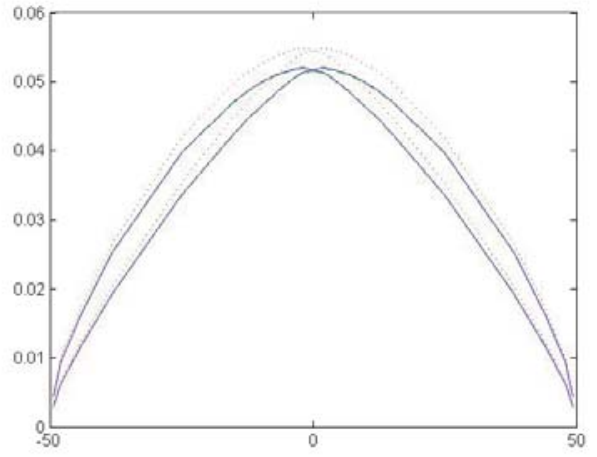

a)

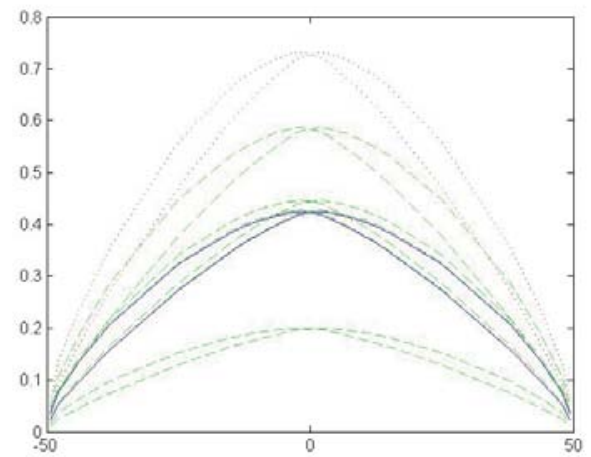

b)

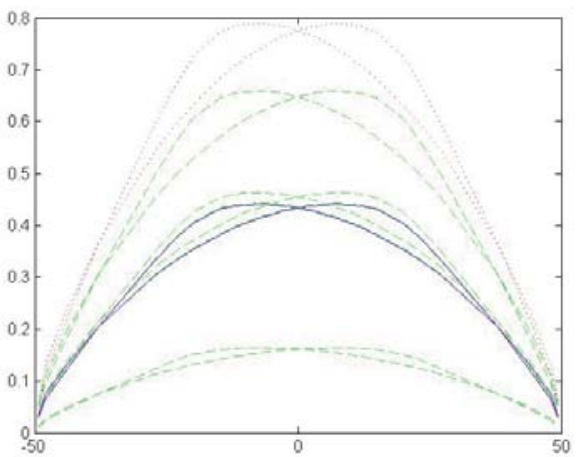

c)

Fig. 4 Heat flow in fibres

Temperature in the centres of fibres is 0 and -52 (the overlapped length is 48 ) for the first two examples and 0 and -70 (the overlapped length is 30 ) for the third example. Temperature increase in the lower temperature fibre as computed in the iteration steps is given in Table 2 and decrease in the higher temperature fibre is opposite. 


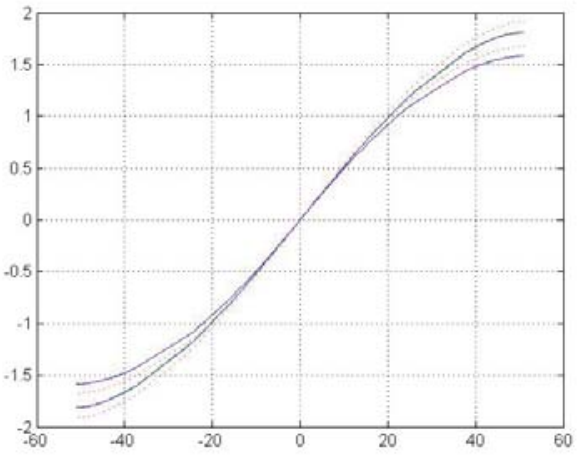

a)

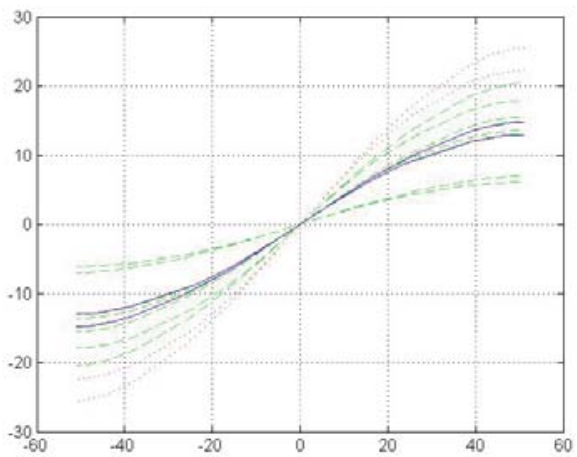

b)

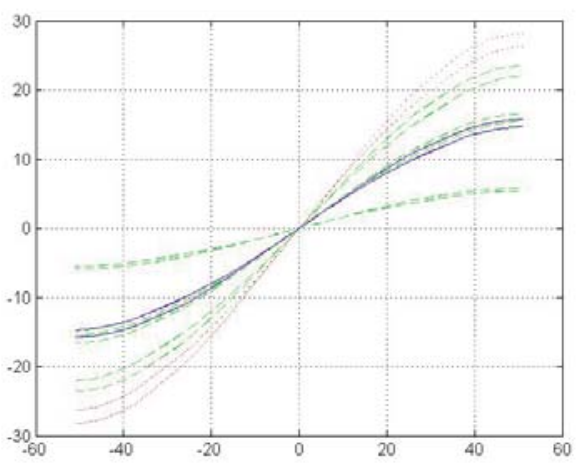

c)

Fig. 5 Temperature along fibres
Temperature change in the centre of fibre

Table 2 with lower temperature

\begin{tabular}{|l|c|c|c|c|c|c|}
\hline & Iter. 1 & Iter. 2 & Iter. 3 & Iter. 4 & Iter. 5 & Iter. 6 \\
\hline Example 1 & 12.2131 & 11.5507 & 11.5870 & 11.5850 & 11.5851 & 11.5851 \\
\hline Example 2 & 12.2131 & 3.3497 & 9.7821 & 7.4480 & 7.1279 & 7.0840 \\
\hline Example 3 & 13.3508 & 2.7760 & 11.1520 & 7.8348 & 7.4899 & 7.4540 \\
\hline
\end{tabular}

\section{Conclusions}

The MCSF is a kind of MFS which uses a fundamental solution and its derivatives to simulate the interaction of short fibres with matrix. Large aspect ratio does not enable to use both source functions in a discrete form and to satisfy the boundary conditions in collocation points. Using the 1D continuous source functions enables to reduce the solution considerably comparing to other known methods.

Source function in heat flow problem is a scalar, in elasticity it is a vector function, however the singularity is similar in both cases and so, the numerical behaviour is also similar [7 and 8]. Because of large gradients in the simulated fields by the interaction, the nodal points defining the continuous source along the fibre axis have to be denser in the parts, i.e. near the ends of the fibres and they have to be irregularly distributed. Numerical experiments have shown that the shape functions defined by NURBS are best suited for the numerical stability and accuracy.

Three numerical examples showing the numerical behaviour of the method on two parallel fibres in an infinite matrix overlapping in some part for a problem of heat transfer. The resulting system of equation is full but not square and is solved by the LS method. Similarly, as it is by BEM, the matrix is full also for the problem containing many fibres, but it can be torn into the interaction of each two fibres and interconnected iteratively, which enables further reduction of the problem [8].

\section{Acknowledgement}

This research was supported by the Slovak Grant Agency VEGA No. 1/1226/12)

\section{References}

[1] NICOLETO, G., BOKUVKA, O., COLLINI, L, KOPAS, P.: Fatigue Resistance in the very High-Cycle Regime. Transaction of FAMENA, vol. 29, No. 1, 2005, pp. 9-16. 


\section{COMMNICOIIONS}

[2] SAGA, M., KOPAS, P., VASKO, M.: Some Computational Aspects of Vehicle Shell Frames Optimization Subjected to Fatigue Life. Communications - Scientific Letters of the Universtiy of Zilina, vol. 12, No. 4, 2010, pp. 73-79.

[3] NISHIMURA, N.: Fast Multipole Accelerated Boundary Integral Equations. Applied Mechanics Review, 55, 2002, pp. 299-324.

[4] BREBBIA, C. A., TELlES, J. C. F., WROBEL, L. C.: Boundary Element Techniques - Theory and Applications in Engineering, Springer Verlag Berlin, New York, 1984.

[5] GOLBERG, M.A., CHEN, C.S.: The method of fundamental solutions for potential, Helmholtz and diffusion problems. Boundary Integral Methods - Numerical and Mathematical Aspect, Ed. M.A. Golberg: Computational Mechanics Publications, Southampton, 1998; pp. 103-176.

[6] KARAGEORGHIS, A., FAIRWEATHER, G.: The Method of Fundamental Solutions for the Solution of Nonlinear Plane Potential Problems, IMA Journal of Numerical Analysis, 9, 1989 pp. 231-242.

[7] KOMPIS, V., KOMPIS, M., KAUKIC, M.: Method of Continuous Dipoles for Modelling of Materials Reinforced by Short Micro Fibres, Engineering Analysis with Boundary Elements 31, 2007, pp. 416-424.

[8] KOMPIS, V., QIN, Q.H., FU, Z. J., CHEN, C.S., DROPPA, P., KELEMEN, M. CHEN, W.: Parallel Computational Models for Composites Reinforced by Short Fibres, Engineering Analysis with Boundary Elements, 36, 2012, pp. 47-52.

[9] PIEGL, L., TILLER, W.: The NURBS Book, Springer-Verlag, New York, $2^{\text {nd }}$ edition, 1997. 